\title{
In Vitro Demonstration of an SpO2-Camera
}

\author{
FP Wieringa ${ }^{1,2}$, F Mastik $^{1}$, RH Boks ${ }^{1}$, A Visscher ${ }^{1}$, \\ AJJC Bogers ${ }^{1}$, AFW Van der Steen ${ }^{1,3}$ \\ ${ }^{1}$ Thorax Centre Erasmus MC, Rotterdam, Netherlands \\ ${ }^{2}$ TNO Science and Industry, Delft, Netherlands \\ ${ }^{3}$ Interuniversity Cardiology Institute of the Netherlands
}

\begin{abstract}
Feasibility of pulse oxigraphy is examined using an invitro phantom, perfused with human blood by a heartlung machine. 29 different oxygen saturation levels, measured with our experimental camera and a clinical pulse oximeter, were matched against laboratory blood gas analysis. Discrete transfer functions between measurements \& laboratory values were derived for camera $\left(f_{\text {CAM-LAB }}\right)$ \& pulse oximeter $\left(f_{\text {PULSE-LAB }}\right) .87$ additional independent pulse oximeter \& camera measurements were subjected to these functions to check reproducibility $\left(R^{2}=0.99\right)$. To demonstrate imaging capacities, a dual reservoir was applied to image venous and arterial samples from 2 patients. Camera-derived vs laboratory arterial values were: $97.0 \%$ vs $99.5 \%$ (pat. 1 , $p H=7.41) \& 97.5 \%$ vs $99.5 \%$ (pat. 2, $p H=7.37$ ). Venous resulst were: $86.5 \%$ vs $74.4 \%$ (pat. $1, p H=7.39$ ) \& $89.1 \%$ vs $86.2 \%$ (pat. 2, pH=7.34). In vitro pulse oxigraphy can visualize regions with different blood oxygenation.
\end{abstract}

\section{Introduction}

Commonly used clinical pulse oximeters produce a numerical value that is derived from photoplethysmographic (PPG) signals at 2 different wavelengths. The obtained oxygen saturation value is obtained from one particular contact site at the body. This can be interpretated as a contact image consisting of only one pixel. As a next step in oxygen-saturation measurement, we have suggested the development of an $\mathrm{SpO}_{2}$ camera, involving the simultaneous non contact detection of a 2-dimensional matrix of spatially resolved PPG signals at 3 different wavelengths $(660,810 \& 940$ $\mathrm{nm})$. The core of this method is to exploit the isobestic behaviour of $\mathrm{Hb}$ versus $\mathrm{HbO}_{2}$ at $810 \mathrm{~nm}$ as a reference to correct for image artifacts like shadows, reflections and pigmentation [1]. In previous work we demonstrated that the PPG signals, needed to achieve this goal, indeed can contactless be derived from the human body, but the obtained signal-to-noise conditions were yet insufficient to calculate meaningful $\mathrm{SpO}_{2}$ values [2]. An approach with alternatingly sampled PPG signals at 2 wavelengths by Humpreys et al. confims that tissue-derived PPGs look feasible for calculating $\mathrm{SpO}_{2}$-images [3].

\section{Methods}

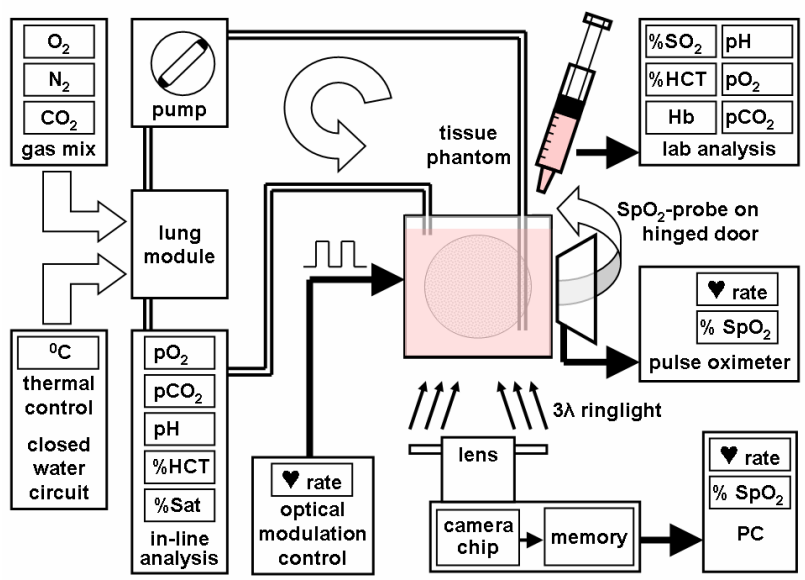

Fig. 1: Setup for triangular comparison of laboratory blood gas analysis, pulse oximetry and camera-derived oxigraphy on an experimental in-vitro phantom. See figure 3 for the results.

Using a variable $\mathrm{O}_{2} / \mathrm{CO}_{2} / \mathrm{N}_{2}$ gas mixture a heart-lung machine with in-line monitoring of $\mathrm{pH}, \mathrm{pO}_{2}$ and $\mathrm{pCO}_{2}$ and derived $\mathrm{SpO}_{2}$ was used to precondition 3 blood pools, aiming for oxygen saturation setpoints of: $98,96,94,92$, $90,88,85,80$ and $75 \%$. The $\mathrm{CO}_{2}$ level was adjusted to keep $\mathrm{pH} 7.35 \pm 0.05$. The conditioned blood circulated through an in vitro phantom consisting of 3 assemblies:

1) A $0.5 \mathrm{~mm}$ thick translucent layer of Delrin ${ }^{\mathrm{TM}}$ (polyoxymethylene with $20 \%$ glassfiber filling) which forms a reasonable optical approximation of human skin at wavelengths $>600 \mathrm{~nm}[4]$. Adjacent to the Delrin ${ }^{\mathrm{TM}}$ layer, a Spectralon ${ }^{\mathrm{TM}}$ target (Labsphere, North Sutton, USA) with > 99\% reflectance from 400-1500 nm, a carbon black target with $<1 \%$ reflectance from $400-1000$ $\mathrm{nm}$ and a printed gray-scale were attached for image quality control purposes. A pulse oximeter probe (Nellcor 
R-15) was mounted on a small hinged door to allow reproducible placement to the Delrin ${ }^{\mathrm{TM}}$ front layer.

2) An electrically controllable spectrally neutral LCDshutter device (Anteryon, Eindhoven, the Netherlands), capable of switching between clear transparant and white diffuse optical state, served as an optical modulator to simulate arterial PPG. The LCD-shutter was fixed at the backside of the translucent Delrin ${ }^{\mathrm{TM}}$ layer. LCD-shutter on/off was controlled by a $50 \%$ duty-cycle $0-5 \mathrm{~V}$ block wave from a 33250A digital waveform generator (Agilent Technologies, Santa Clara, California USA).

3) A small blood reservoir, consisting of a CLS3055 polystyrene cell culture flask (Corning, Schiphol-Rijk, the Netherlands) positioned at the backside of the LCDshutter. Via two luer lock connectors this flask was fitted to the artificial blood circulation.

The above described translucent Delrin layer, LCDshutter and blood reservoir as well as the camera and pulse oximeter probe at the hinged door, were placed together in a light tight enclosure with a side acces light tight hatch door. For each setpoint, a monochrome CMOS-camera (Fillfactory, Belgium) with apochromatic lens (at $22 \mathrm{~cm}$ distance from the phantom) and $3 \lambda$-LEDringlight (100LEDs $\left.\lambda^{-1}\right)$ sequentially recorded $3 \lambda$-moviesets of the phantom at $60,80,100$ and 120 beats $\mathrm{min}^{-1}$, followed by reflectance mode pulse oximetry (Nellcor N200). Each acquisition at 120 beats min-1 was followed by laboratory blood gas analysis (Radiometer ABL725), which formed the gold standard. All in-line analysis instruments were only used to verify whether setpoints had reached a stable value.

Pulse oximeter values for oxygen saturation were directly read from the display. As for the camera, each $3 \lambda$-set of ROI-pixel-time-traces was processed to calculate the blood oxygen saturation related ratio-ofratios value. First of all, for each wavelength and all ROIpixel-time-traces $\left(S_{\lambda}\right)$ the difference between the mean high plateau and mean low plateau value of the block wave (AC component) was determined by $A C_{\lambda}=S_{\lambda, \text { high }}-S_{\lambda, \text { low }} \quad$ resulting in distinct AC "peak-tovalley" values for $\lambda=660,810$ and $940 \mathrm{~nm}$.

The DC component $D C_{\lambda}$ was defined as the mean value of $S_{\lambda}$ over all 160 recorded frames.

These values were used to calculate the so-called ratioof-ratios R for $660 \mathrm{~nm}$ and $940 \mathrm{~nm}$ :

$$
R_{660 / 940}=\frac{\frac{A C_{660}}{D C_{660}}}{\frac{A C_{940}}{D C_{940}}}
$$

We applied the ratio-of-ratios method to likewise find:

$$
R_{660 / 810}=\frac{\frac{A C_{660}}{D C_{660}}}{\frac{A C_{810}}{D C_{810}}} \text { and } R_{940 / 810}=\frac{\frac{A C_{940}}{D C_{940}}}{\frac{A C_{810}}{D C_{810}}}
$$

The values obtained at all setpoints for the ratio-of ratios $\overline{R_{660 / 940}}, \overline{R_{660 / 810}}$ and $\overline{R_{810 / 940}}$ respectively, were compared to the pulse oximeter $\mathrm{SaO}_{2}$ readings and to the lab-derived $\mathrm{SO}_{2}$ values.

By subjecting all individual ROIs for which a valid heart rate was determined to $\mathrm{f}_{\mathrm{CAM}-\mathrm{LAB}}$ and assigning a false color scale to the results, oxigrams obtained at several oxygenation settings could be superimposed upon the oxygen independent $810 \mathrm{~nm}$ greyscale image.

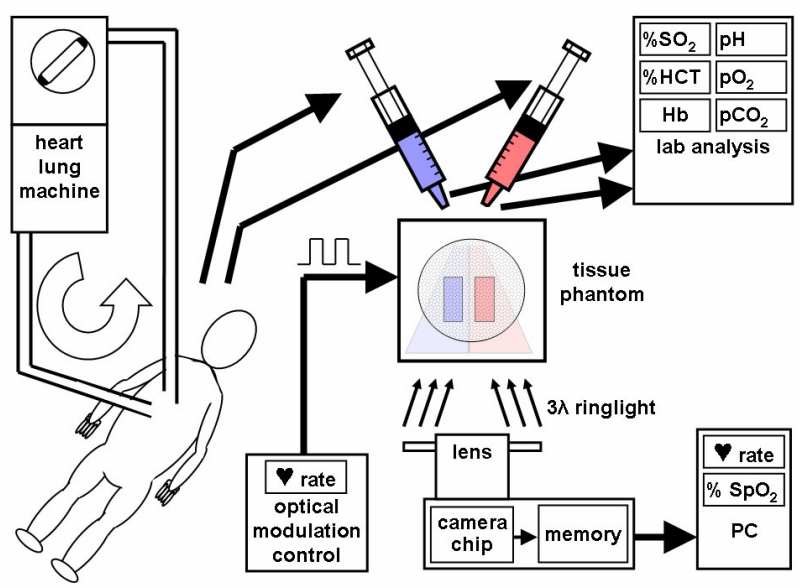

Fig. 2: Additional experiment with dual chamber phantom using venous \& arterial blood samples from patients, to demonstrate $\mathrm{SpO}_{2}$-imaging. For the resulting $\mathrm{SpO}_{2}$-image see figure 4 .

An additional experimental setup to demonstrate the cameras' imaging capacities is shown in figure 2 . The phantom was equipped with a dual-chambered blood reservoir and modified so that two discrete PPG regions resulted. Venous and arterial blood samples $(30 \mathrm{ml}$ each $)$ were collected from stable patients $(\mathrm{n}=2)$ during extracorporal perfusion. $25 \mathrm{ml}$ of each sample was injected in the venous, resp. arterial blood reservoir and 5 $\mathrm{ml}$ was immediately analyzed in the laboratory.

\section{Results}

The setup of figure 1 produced camera-derived results having monotone and reproducible relations with pulse oximetry and laboratory values (see upper halve fig. 3).

Pulse oximeter readings decreased about a factor 4 steeper than laboratory values when lowering the saturation setpoint (see lower halve fig. 3). 

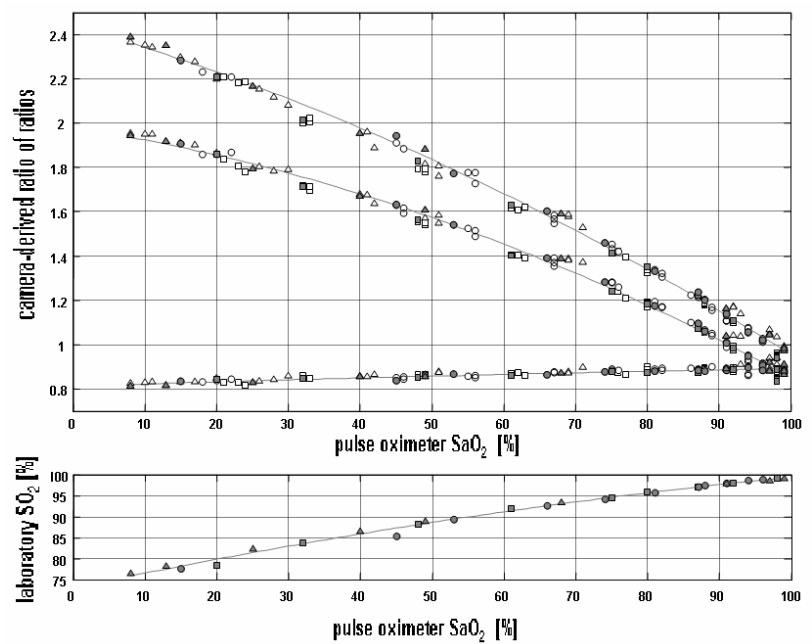

Fig. 3: The top graph shows the relation between camera derived data and pulse oximetry. The vertical axis represents the dimensionless values of $\overline{R_{660 / 940}}$ (top trace), $\overline{R_{660 / 810}}$ (middle trace) and $\overline{R_{810 / 940}}$ (bottom trace). The horizontal axis represents pulse oximeter $\mathrm{SaO}_{2}$ readings. Third-order least-squares spline approximation is fitted through 29 observations of $\overline{R_{660 / 940}}$, $\overline{R_{660 / 810}} \& \overline{R_{810 / 940}}$ having corresponding lab values. The lower graph shows phantom transfer function $\mathrm{f}_{\mathrm{PULSE}-\mathrm{LAB}}$. Here also pulse oximeter $\mathrm{SaO}_{2}$ readings form the horizontal axis, but the vertical axis represents the "gold standard" lab-derived $\mathrm{SO}_{2}$ value. The 3 different blood pools are indicated using circular, triangular and square markers, whereas filled-up markers indicate points for which also a lab value exists.

The $R_{660 / 940}$ pixel matrix filmed with the figure 2 setup was subjected to $\mathrm{f}_{\mathrm{CAM}-\mathrm{LAB}}$ and a false color scale value (red $=$ high saturation; blue $=$ low saturation) was assigned to each ROI-pixel with sufficient PPG amplitude. The resulting images (proposed term "pulse oxigrams") clearly revealed distinct arterial and venous phantom regions (see figure 4).

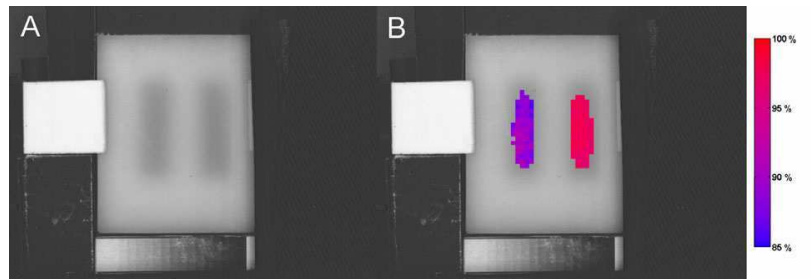

Fig. 4: NIR 810nm reference (A) and pulse oxigram (B) of a dual chamber phantom filled with human blood (L venous; $R$ arterial). The naked eye could hardly see through the diffuser.

Camera-derived arterial saturations vs lab results were: $97.0 \%$ vs $99.5 \%$ (pat $1, \mathrm{pH}=7.41$ ) \& $97.5 \%$ vs $99.5 \%$ (pat2, $\mathrm{pH}=7.37$ ). Venous results were: $86.5 \%$ vs $74.4 \%$ (pat1, $\mathrm{pH}=7.39$ ) \& $89.1 \%$ vs $86.2 \%$ (pat2, $\mathrm{pH}=7.34$ ).

\section{Discussion and conclusions}

The processing algorithm was applied to the whole image, discriminating PPGs well against the background.

Within the arterial phantom region, deviations in oxygen saturation (camera-derived minus laboratory value) were relatively small $(-2.5 \% \&-2.0 \%)$, and the camera slightly underestimated saturation. Within the venous phantom region, however, the camera overestimated saturation $(+12.1 \% \&+2.9 \%)$.

The reason for the relatively large deviation between the camera-derived and laboratory results for the venous region of patient 1 may be that here venous saturation was beyond the range for which transfer function $\mathrm{f}_{\mathrm{CAM} \text { - }}$ ${ }_{\mathrm{LAB}}$ had been derived. However, results are also likely to be influenced by geometrical differences in the dual chamber phantom compared to the single chamber phantom as used to derive $\mathrm{f}_{\text {CAM-LAB }}$.

Pulse oxigraphy using the ratio-of-ratios method can visualize differences in geometrical distribution of blood oxygen levels in-vitro, but needs further improvements.

\section{Acknowledgements}

This work was sponsored by TNO Quality of Life and TNO $\mathrm{O}_{2}$-View $\mathrm{BV}$, both based in Leiden, the Netherlands. Custom mechanical constructions were made by Mr. L. Bekkering and Mr. G. Springeling, also support on electronics was given by Mr. J. Honkoop and Mr. W. van Alphen (Biomedical Engineering Thorax Centre, Erasmus MC). We thank Mr. J. Sweep and Mr. A. Klippel (Biomedical Instrumentation Sophia Childrens Hospital, Erasmus MC), all members of the perfusionistsgroup and all workers of the Thorax Centre Clinical Lab.

\section{References}

[1] Wieringa FP. Imaging apparatus for displaying concentration ratios. Patent WO 01/15597 A1 2001.

[2] Wieringa FP, Mastik F, ten Cate FJ, Neumann HAM and van der Steen AFW. Remote non-invasive stereoscopic imaging of blood vessels: First in-vivo results of a new multispectral contrast enhancement technology. Annals of biomedical engineering 2006;34(12)1870-187

[3] Humphreys K, Ward T and Markham C. Noncontact simultaneous dual wavelength photoplethysmography: A further step toward noncontact pulse oximetry. Review of scientific instruments 2007, 78(044304);1-6

[4] Okamoto Ugnella A, Öberg PÅ, The optical properties of the cochlear bone. Medical Engineering \& Physics 1997;19(7):630 - 636

\section{Address for correspondence}

F.P. Wieringa, e-mail f.wieringa@erasmusmc.nl Erasmus MC, Biomedical Engineering, Office Ee 2302 P.O. Box 2040, 3000 CA Rotterdam, Netherlands 